\title{
Estudio de la influencia del confinamiento debido a la COVID-19 en padres, alumnado y profesorado en ESO y FP
}

\author{
Rosa Pérez ${ }^{1}$, Claudia Villalonga², Oresti Baños ${ }^{1}$, Alberto Guillén ${ }^{1}$ \\ ${ }^{1}$ Departamento de Arquitectura y Tecnología de Computadores, Universidad de Granada \\ Granada, España \\ \{aguillen, oresti\}@ugr.es \\ 2 Escuela Superior de Ingeniería y Tecnología, Universidad Internacional de La Rioja \\ Logroño, España \\ claudia.villalonga@unir.net
}

\begin{abstract}
Resumen. En este artículo se describe una investigación educativa en la que se estudia cómo afecta el confinamiento a alumnos, padres y profesores de secundaria, bachillerato y formación profesional. Dicho confinamiento surgió para evitar la propagación de la pandemia originada por la enfermedad COVID19 y provocó que los alumnos y docentes se viesen obligados a tener clases online. Esto ha supuesto grandes retos y dificultades que han afectado a su estrés, motivación y aprendizaje. Se ha realizado un análisis sobre cómo se está llevando a cabo la docencia virtual y se ha comprobado que no ha tenido una organización eficiente, por lo que se ha elaborado una propuesta de mejora para situaciones similares futuras.
\end{abstract}

Palabras Clave: confinamiento, metodología docente, alumnado, COVID-19

\begin{abstract}
This work presents a research on the effects of confinement on students, families and teaching staff at high school. The confinement was originated within the context of the COVID-19 pandemic and it forced all the individuals to rapidly transition from in-class to online teaching. This new teaching context has made the situation even more stressful and challenging for both students and teachers. The research described in this article questions how online teaching has been developed during the pandemic. In addition to that, the paper proposes some recommendations for the relevant stakeholders to improve the teaching organisation and procedures for a future confinement.
\end{abstract}

Keywords: confinement, teaching methodology, students, COVID-19 


\section{Introducción}

España se enfrenta a una situación muy complicada debido a la pandemia COVID19, en la que los jóvenes se han visto obligados a no poder salir de casa y a recibir las clases online. Permanecer todo el día en casa privados de clases, actividades con sus amigos, juegos y ejercicios al aire libre, puede derivar en un aumento del estrés, irritabilidad o ansiedad. Todo ello genera que necesiten llamar más la atención en casa causando multitud de conflictos domésticos. Es una situación nueva y extraña para todos, por lo que hay que tener mucho cuidado sobre cómo gestionarla. La acción de los padres es fundamental, ya que deben gestionar las emociones de sus hijos y proponerles alternativas para canalizar sus emociones. Es importante mantener rutinas y horarios [1].

Además, las clases online conllevan multitud de dificultades y problemas. Por un lado, tanto los profesores como los alumnos no suelen tener una gran experiencia impartiendo y recibiendo docencia de forma virtual. Por otro lado, no todas las familias tienen acceso a Internet y en muchos casos los jóvenes no disponen de un ordenador propio [2]. Hay que tener en cuenta que hay personas que se juegan la obtención de un título, como el de E.S.O. (Educación Secundaria Obligatoria), Bachillerato o FP (Formación Profesional) y otras que ven peligrar su acceso a la universidad. Todos los factores mencionados pueden generar estrés y desmotivación. A todo ello hay que añadir que, además de la brecha digital, hay familias que tienen problemas muchos más graves como puede ser el desempleo o la posible pérdida de la vivienda. Por ello es de vital importancia que las administraciones públicas gestionen de la mejor forma posible estos casos proporcionando ayuda económica, educativa y psicológica a las familias más desfavorecidas.

Un problema que se plantea en todos los niveles educativos es la evaluación. El ministerio de educación de España acordó con las diferentes comunidades autónomas que todos los alumnos de ESO, FP y Bachillerato pasasen de curso salvo casos muy excepcionales [3]. En la Instrucción del 23 de abril de 2020 se detalla cómo deben actuar los docentes ante la evaluación de los alumnos. Aquí se especifica que sólo se tendrán en cuenta los dos primeros trimestres y que el tercero únicamente se tendrá en cuenta en la evaluación del estudiante para mejorar, en ningún caso perjudicará al alumno. Además, no es necesario impartir los elementos del currículum que continúen en el año académico 2020/2021. En todo momento se antepondrá la salud emocional y física del alumno y se tendrá en cuenta su contexto. Se primará el repaso y la recuperación de los dos primeros trimestres. No obstante, en Educación Secundaria, Bachillerato y Formación Profesional, el profesorado no está muy a favor de esta medida [4]. Los docentes son partidarios de evaluar los objetivos mínimos del currículum con los medios que tienen a su disposición. Sin embargo, la evaluación virtual es muy compleja, ya que es difícil detectar la autoría de las tareas y exámenes. Por todo ello, es necesario que haya responsabilidad por parte de la Administración, con dotación de recursos para satisfacer las necesidades de las familias más desfavorecidas; responsabilidad de los profesores, ajustando las programaciones y unidades didácticas conforme a la Instrucción del 23 de abril; y responsabilidad de los alumnos y sus familias ante esta situación.

Antes del confinamiento la mayoría de adolescentes ya pasaban más tiempo conectados a Internet que estudiando según una investigación realizada en España, 
Reino Unido y Estados Unidos [5], cuyos resultados se muestran en la Figura 1. No obstante, este hecho posiblemente se incremente con el estado de confinamiento con sus correspondientes repercusiones. Sin embargo, no es aconsejable que los padres sean demasiados exigentes, sino que deben aplicar "el principio de la amabilidad y la firmeza", conseguir un balance entre autoridad y generosidad, ganándose el respeto de sus hijos desde la comprensión. Además, los menores deben sentirse protagonistas de la situación, siendo los padres los que les ayuden a ver que su papel es muy importante a la hora de controlar la pandemia. A todo ello hay que añadir que los padres pueden aprovechar esta situación para hacerles ver el uso correcto de las nuevas tecnologías, como puede ser el contacto con otros familiares o tours virtuales alrededor de todo el mundo [1].

\section{Los menores de Secundaria dedican 4 horas más al año a Internet que a su tiempo de estudio}

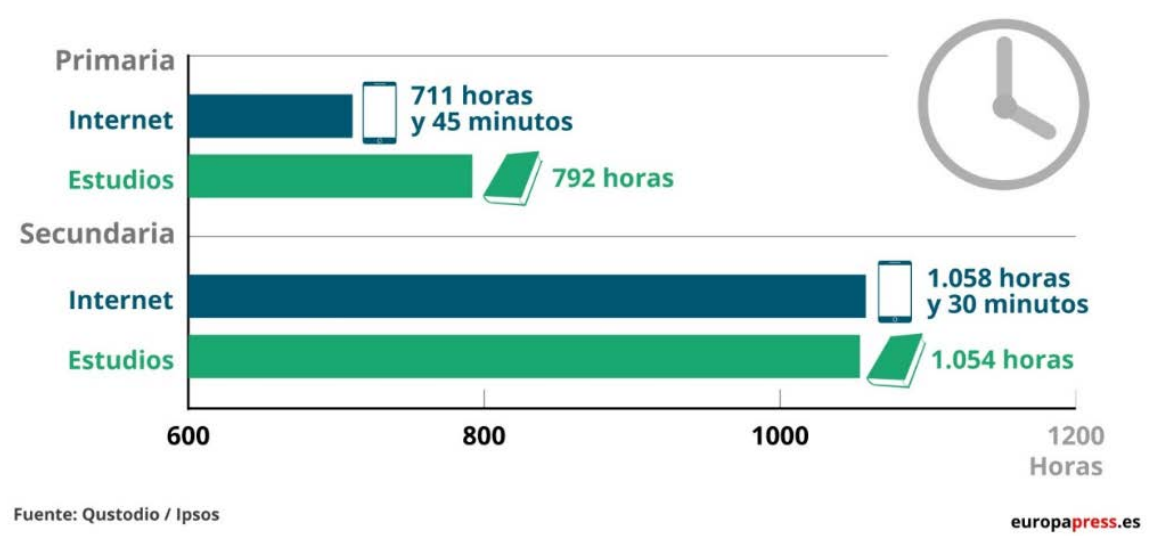

Figura 1. Comparativa entre el tiempo que los adolescentes dedican a Internet y a estudiar (Fuente: Europapress, 2019)

Hay muchos estudiantes que piensan que la docencia online es un caos, ya que desde la Administración educativa se deberían establecer directrices claras sobre cómo actuar en los diferentes centros. Sin embargo, éstas han dejado caer todo el peso de la responsabilidad en los docentes, generando un gran caos y descontrol en el uso de diversas herramientas, que lejos de ayudar a los estudiantes, han aumentado su estrés y desprecio hacia las clases virtuales. A ello se suma el hecho de que muchos estudiantes piensan que la carga académica es excesiva para la situación vivida. Un aspecto positivo es que se está fomentando la capacidad de aprender de forma autodidacta, no obstante, se debería liberar un mayor volumen de contenido académico en la red, para que fuese de dominio público y todos los estudiantes pudiesen consultarlo independientemente de su economía. Además, la inmensa mayoría de las plataformas de aprendizaje utilizadas pertenecen a empresas privadas, por lo que en cierta medida se está "privatizando" la educación [6].

Como se ha comentado en el capítulo anterior, la actividad física es fundamental para un correcto desarrollo del individuo. El confinamiento puede afectar gravemente a la salud mental de los adolescentes [7] y puede generarles depresión y un doble 
aislamiento [8]. Los menores necesitan realizar ejercicio para el buen desarrollo de su sistema nervioso, ya que los estímulos permiten desarrollar el aprendizaje. El hecho de estar encerrados es antinatural y puede generar graves consecuencias.

Por todo ello es muy importante realizar una investigación acerca de la docencia online, las herramientas virtuales usadas, la competencia que tienen los profesores y alumnos con dichas tecnologías, el estrés, la motivación, la importancia del factor humano, el deporte realizado en casa, las horas de estudio o la actividad más realizada durante el día.

Dejando a un lado todos los problemas que conlleva el confinamiento, hay que buscar soluciones. Las clases virtuales también ofrecen una serie de ventajas, ya que hay diferentes plataformas que permiten realizar vídeo llamadas entre el profesor y sus estudiantes para impartir las clases online, ofreciendo la posibilidad de grabar dichas clases para que los alumnos que no han podido asistir las vean en diferido. Además, los alumnos pueden centrarse completamente en las explicaciones del profesor, aprovechando las clases grabadas para consultar contenidos, dudas a posteriori o tomar algún apunte. Es importante tener en cuenta que durante las clases presenciales hay una gran comunicación implícita en el lenguaje corporal y los gestos faciales. Es importante realizar multitud de preguntas por parte del profesor para asegurarse de que los alumnos han comprendido correctamente los contenidos impartidos. También hay que definir de manera clara y concisa las tareas que debe realizar el alumnado para que no haya lugar a confusión [9]. Por ello se evaluará cómo son actualmente las clases presenciales y se realizará una propuesta de mejora a partir de la información obtenida.

\section{Metodología}

\subsection{Población y muestra}

Para valorar cuáles son los instrumentos de recogida de información óptimos para esta investigación, hay que tener en cuenta la población (conjunto de individuos que poseen una característica determinada) y la muestra (subconjunto significativo de la población) [10]. Tras consultar la colaboración de varios centros de la provincia de Málaga y Granada, el I.E.S. María Zambrano aceptó su participación en la investigación propuesta. Por lo tanto, el estudio está compuesto por 3 poblaciones (profesores, alumnos y padres), cuya muestra está formada por los individuos que rellenaron el consentimiento informado. Además, se dividen en tres estratos (ESO, Bachillerato y FP).

Dicho centro se encuentra situado en la localidad de Torre del Mar, en la costa oriental de la provincia de Málaga, entre el barrio Las Malvinas y Viña Málaga. Gran parte del alumnado reside en dichos barrios, otros en El Tomillar y un número importante de estudiantes viene de pequeñas poblaciones próximas a dicha localidad, como son Almayate o Benajarafe. En cuanto a las familias, hay una gran diversidad, desde niveles sociales bajos hasta clase media alta. De acuerdo al proyecto educativo del centro [11], una inmensa mayoría considera que sus hijos continuarán su formación 
en estudios universitarios y postobligatorios. La mayoría de los estudiantes se encuentran entre los 12 y 22 años, en función del curso que estén realizando. No obstante, hay alumnos estudiando Formación Profesional con edades más avanzadas. La mayoría de las personas que se encuentran realizando sus estudios en el centro son adolescentes, en cuyo período de la vida tienen lugar cambios importantes tanto psicológicos como físicos y fisiológicos. Además, es en esta etapa cuando cambian del pensamiento concreto al formal o abstracto. No obstante, este cambio es diferente para cada persona lo cual se traduce en una gran diversidad en el aula.

Se trata de un centro bilingüe en el que se imparten clases en español e inglés. Las enseñanzas que se ofertan son ESO, Bachillerato, FP y P.M.A.R. (Programas para la Mejora del Aprendizaje y el Rendimiento) y A.T.A.L. (Aula Temporal de Adaptación Lingüística). El instituto también cuenta con estudiantes que tienen necesidades especiales de apoyo educativo (N.E.A.E.), en su mayoría por problemas de aprendizaje. Por ello, el centro cuenta con un profesional de pedagogía terapéutica.

El centro cuenta con multitud de programas internacionales como "Comenius", "Bachillerato Internacional" y "Erasmus". También se ofrecen proyectos culturales como "Programa Aldea", "Forma Joven" o "Escuela: Espacio de Paz" [11].

\subsection{Recogida de información}

A continuación, se comenta cada instrumento de recogida de información y se eligen aquellos que se adaptan mejor al estudio concreto. Concretamente se utiliza el cuestionario para recoger la información de las tres poblaciones (uno para cada uno de ellas). Asimismo, se emplean entrevistas personales que permiten recabar percepciones y opiniones directas de los alumnos, profesores y padres/madres.

Son numerosos los estudios realizados sobre el rendimiento académico usando el cuestionario [12, 13]. Por lo tanto, en este trabajo se recaban datos a través de tres cuestionarios diferentes: uno para los padres/madres; otro para los alumnos; y un tercero para los docentes. Con ello se consigue contrastar la información aportada por el estudiante y el entorno, permitiendo extraer conclusiones mucho más certeras, para poder realizar un plan de mejora con mayor nivel de éxito.

Para la redacción de los ítems que forman este instrumento hay que tener en cuenta una serie de instrucciones [14, 15, 16]:

- Deben ser claros y simples para que puedan ser comprendidas por todos los sujetos.

- Deben ser muy breves. Hay que tener en cuenta que los adolescentes no suelen tener una gran atención lectora, por lo que las preguntas no deberán superar las 25 palabras.

- Deben obviarse las palabras que lleven a interpretaciones confusas. Para que una pregunta sea precisa las personas encuestadas tienen que comprenderla del mismo modo. Las especificaciones temporales o numéricas deben ser concretas, como pueden ser "dos veces al mes" o "todas las semanas". Hay que evitar expresiones como "a menudo", "de vez en cuando" o "casi nunca".

- Deben ser neutrales para que no influyan en la respuesta de los encuestados y no se creen sesgos que impidan saber la verdadera opinión de los sujetos. 
- Deben eludirse aquellas formuladas en tono negativo ya que son más complicadas de entender.

- Deben evitarse aquellas que en las que el encuestado deba hacer un gran uso de memoria. Si es absolutamente necesario, se deben realizar cuestiones focalizándose en los sucesos más próximos, ofreciendo una serie de respuestas exhaustivas.

- Deben incluir una única idea para no dar lugar a confusión.

- Deben estar adecuadas al individuo al que se dirigen.

El contenido de los ítems es muy importante, pero también lo es su orden:

- Las cuestiones tienen que ir de menos a más comprometidas.

- Las cuestiones deben seguir una secuencia lógica de temas.

- Las primeras preguntas deben ser simples.

- Los ítems de identificación suelen ir al principio y deben garantizar el anonimato.

- Las cuestiones cuya respuesta suponga seleccionar una serie de cuadros deben colocarse de modo que no resulten fatigosas.

Teniendo en cuenta estos elementos listados y los objetivos del estudio, se definen las siguientes variables que se medirán a través del cuestionario: grado de satisfacción de las clases virtuales, grado de satisfacción de las clases presenciales, grado de satisfacción de las medidas académicas adoptadas por el Ministerio de Educación, motivación académica, experiencia en plataformas virtuales, deporte, estudio y entorno en el hogar.

Los cuestionarios deben ofrecer la posibilidad de responderse en menos de 15 minutos para evitar la fatiga del encuestado. Se debe proporcionar absoluta confidencialidad. Además, se debe realizar una prueba piloto para comprobar su correcto funcionamiento y realizar posibles mejoras.

Otra herramienta utilizada para recoger información ha sido la entrevista. Gracias a este instrumento es posible recoger aspectos personales directamente del individuo bajo estudio, sobre todo en el caso de que esta sea abierta. Además, puede llegar a ser más eficaz que el cuestionario ya que la información que se obtiene del sujeto es más profunda [17]. Debido a la situación de confinamiento no es posible realizar las entrevistas de forma presencial, por lo que se llevan a cabo a través de "Google Meet".

De los tres tipos de entrevista (estructurada, semiestructurada y no estructurada), se escoge la entrevista semiestructurada para realizar esta investigación, ya que ofrece más flexibilidad que la estructurada y las preguntas pueden ajustarse a las personas bajo estudio. Esto permite la posibilidad de motivar al entrevistado, evitando ambigüedades y resolviendo posibles dudas. Además, la entrevista no estructurada tiene la desventaja de que puede generar falta de información necesaria para la investigación y los sujetos se pueden desviar en exceso del tema en cuestión.

Para dicho tipo de entrevista es importante seguir una serie de pautas [18]:

- Tener una guía con cuestiones agrupadas por temas.

- Escoger un espacio sin ruidos que dificulten la entrevista.

- Aclarar a la persona entrevistada el porqué de la misma.

- Recabar los datos personales necesarios para la investigación. 
- Tener una actitud sensible y receptiva.

- Adaptar las preguntas y el orden al entrevistado según esté yendo la entrevista.

- No detener al entrevistado y dar libertad para que hable de otros temas.

\section{Resultados y propuesta de mejora}

A partir de los resultados obtenidos después de analizar los datos registrados se determina que la inmensa mayoría de los alumnos quieren que termine el confinamiento, sobre todo los de FP. Se puede ver que hay algún alumno de bachillerato que no quiere que termine el confinamiento, quizás al verse encerrado pueda tener mayor capacidad de concentración, mientras que la docencia impartida en FP es muy práctica y quizás no tiene mucho sentido impartir determinados contenidos de forma virtual.

En cuanto a las clases virtuales, los estudiantes de FP y bachillerato no están contentos con ellas, y a pesar de que hay alumnos de bachillerato a los que tampoco les gustan las clases presenciales, ambos grupos coinciden en que prefieren clases presenciales antes que clases virtuales. No obstante, a los alumnos de ESO sí que les gustan las clases virtuales y las prefieren antes que las presenciales. Sin embargo, hay alumnos de educación secundaria que afirman que sus clases virtuales son algo caóticas, pero no necesariamente por el hecho de realizarse online. Todo apunta a que a muchos de estos alumnos les gustaría tener clases virtuales siempre que estuviesen bien planteadas. Posiblemente estas clases virtuales no se estén llevando a cabo de forma correcta, ya que en los resultados obtenidos se observa que los profesores no tienen experiencia previa en docencia online. Por otro lado, todas las madres cuyos hijos cursan ESO, prefieren que sus hijos tengas clases presenciales antes que virtuales, mientras que hay madres, cuyos hijos cursan bachillerato, que prefieren las clases virtuales en lugar de las presenciales. Esto puede deberse a que los estudiantes de ESO son todavía bastante jóvenes e inmaduros para seguir la docencia online según la opinión de sus padres. En el caso de los alumnos de bachillerato, con alumnado que está preparando la selectividad, sus padres consideran que hay clases en las que no se dan correctamente los contenidos y que aprovechan mejor el tiempo en casa. Por último, los docentes tienen muy claro que prefieren las clases presenciales con una destacada mayoría del 95\%.

Si se analizan los resultados relativos al estrés, se puede observar que los alumnos de FP y bachillerato están bastante estresados, especialmente estos últimos, quizás debido a la prueba de acceso a la universidad. No obstante, los alumnos de ESO no tienen la percepción de estar estresados. Una de las posibles causas puede ser que los alumnos de ESO consideran que las tareas académicas que deben realizar se adecuan a tiempo y contenidos, mientras que los alumnos de bachillerato y FP piensan lo contrario. Además, el estrés está muy relacionado con la motivación. De hecho, podemos ver que los alumnos de ESO están más motivados que los otros dos grupos. Las madres también piensan que los alumnos de bachillerato están más estresados que los de ESO. Hay profesores que se sienten más estresados que antes del confinamiento y otros que se sienten menos estresados. Esto puede deberse a que algunos profesores 
no se estén preocupando demasiado por la docencia online, mientras que otros sí están haciendo un esfuerzo extra por adaptar la docencia.

De los resultados de las encuestas, la preferencia de las clases virtuales con la idea personal de que están aprendiendo mucho más durante el confinamiento se demuestra muy correlacionada. El grado de aprendizaje también este correlacionado con la motivación y el tiempo de deporte realizado semanalmente, afianzando el hecho de que el deporte está relacionado con el rendimiento académico. La motivación personal está relacionada con la motivación recibida por parte del profesor, siendo fundamental en la situación vivida por los alumnos. En el análisis de las correlaciones del cuestionario realizado a las madres, se observa que los alumnos que realizan deporte están más motivados. En cuanto a los docentes, se aprecia que el nivel de estrés de los profesores está altamente relacionado con el seguimiento individual que están realizando de sus alumnos y con el hecho de que echen de menos interactuar presencialmente con sus alumnos en clase. El fin del confinamiento está muy relacionado con el tiempo que dedican a la docencia y con querer dar clases presenciales. En todos los casos, el fin del confinamiento está relacionado con la preferencia de clases presenciales.

\subsection{Propuesta de mejora}

Una vez analizados los resultados obtenidos tras la realización de las diversas entrevistas y cuestionarios, se hace evidente que las clases virtuales no han tenido una organización eficiente. Por ello es necesario realizar una mejora para casos venideros.

Como primera medida, se sugiere que cada centro debe establecer una única plataforma virtual, ya que para los alumnos resulta muy confuso utilizar distintas plataformas, según los datos obtenidos.

Una vez elegida la plataforma, es necesario habituar a los alumnos, profesores y padres a su uso. Para ello se formarán a los docentes en la plataforma seleccionada. A continuación, los profesores deberán impartir una pequeña parte de cada unidad didáctica usando dicha plataforma. El centro educativo debe formar a los alumnos en el uso de dicha plataforma además de considerar la impartición de talleres para los padres.

Cuando comiencen las clases online, ya sea por causa de fuerza mayor o por decisión del centro, todos los profesores deberán impartir su docencia usando las plataformas anteriores. Además, tendrán que explicar los contenidos mediante vídeo llamadas con sus alumnos exclusivamente. Se recomienda la grabación de éstas para que los alumnos que no puedan asistir tengan la opción de seguirlas en diferido. Durante estas vídeo llamadas, los alumnos podrán plantear diferentes preguntas para que el profesor las resuelva. Se podrán mandar tareas para realizarlas en directo o a posteriori. El Gobierno Regional de cada Comunidad Autónoma, deberá tomar la decisión sobre el número de horas impartidas a la semana a través de vídeo llamadas, decidiendo si deben seguir con el horario de las clases presenciales o si deben impartir una clase semanal para cada asignatura. En el segundo caso, entre dos asignaturas consecutivas debe haber un margen de dos horas y cada una de ellas puede tener una duración máxima de una hora y media. Los treinta minutos sobrantes se dedicarán a las relaciones interpersonales en las que los alumnos podrán conversar entre ellos o con el profesor si 
éste lo desea. A todo ello hay que añadir que los inspectores podrán acceder a dichas reuniones.

La evaluación virtual es compleja, ya que es difícil controlar la autoría de las tareas y exámenes. No obstante, es posible concertar reuniones virtuales con los estudiantes para hacer exámenes orales o realizar diversas preguntas sobre tareas y trabajos determinados y comprobar si se ha realizado o no plagio.

Por último, a comienzos del curso escolar se deben tener listas las programaciones y unidades didácticas presenciales y virtuales, como plan de contingencia ante un posible rebrote o algún posible evento inesperado.

\section{Conclusiones}

Este trabajo ha presentado una investigación sobre la percepción de las clases que se han realizado a distancia durante el confinamiento que ha sido originado por la pandemia de la COVID-19. La información ha sido recopilada mediante cuestionarios y el formato de entrevista por videoconferencia. El sentimiento de caos y estrés se ve reflejado en los resultados de dichos instrumentos de medida aunque hay que ser conscientes del estrés intrínseco de la situación. Tanto docentes como familias y parte del alumnado consideran que la docencia online no es satisfactoria y en este trabajo se sugieren algunas pautas que podrían mejorar la satisfacción.

\section{Referencias}

1. Borraz, M (20 de marzo de 2020). Así afecta a los niños el confinamiento: pautas para gestionar la cuarentena con hijos y no tirarte de los pelos. El diario. Recuperado de: https://www.eldiario.es/nidos/Confinamiento-ninos_0_1007549790.html

2. Mas, A. (14 de abril de 2020). La educación online no llega a todos los alumnos. La Vanguardia. Recuperado de:

https://www.lavanguardia.com/vida/formacion/20200414/48495012419/educacion-onlineno-llega-todos-alumnos.html

3. Torres, A. (15 de abril de 2020). El Gobierno acuerda con las autonomías que todos los alumnos pasen de curso, salvo casos muy excepcionales. El País. Recuperado de: https://elpais.com/sociedad/2020-04-15/el-gobierno-acuerda-con-las-autonomias-elaprobado-general-salvo-casos-muy-excepcionales.html

4. Trujillo, F. T. \& Fernández, M. (2020). Escenarios de evaluación en el contexto de la pandemia por la COVID-19: la opinión del profesorado.

5. Los niños españoles de 12 a 17 años pasan más horas al año en Internet que en el colegio, según un estudio. (22 de octubre de 2019). Europapress. Recuperado de: https://www.europapress.es/sociedad/educacion-00468/noticia-ninos-espanoles-12-17anos-pasan-mas-horas-ano-internet-colegio-estudio-20191022120544.html

6. Comisión Ejecutiva General (2020). El sistema educativo en la crisis del COVID19. Frente de Estudiantes. Recuperado de: https://www.frentedeestudiantes.es/wpcontent/uploads/2020/04/Informe_COVID19.pdf 
7. Pérez-Bryan, A. (28 de marzo de 2020). Salud mental en niños y adolescentes: hasta dónde les afecta el encierro. Diario Sur. Recuperado de: https://www.diariosur.es/malagacapital/salud-mental-ninos-20200324105117-nt.html

8. Forminaya, C. (27 de marzo de 2020). Adolescentes que no salen de su cuarto: doblemente aislados durante el confinamiento. ABC. Recuperado de: https://www.abc.es/familia/padreshijos/abci-adolescentes-no-salen-cuarto-doblemente-aislados-durante-confinamiento202003270217_noticia.html?ref=https\%3A\%2F\%2Fwww.google.com\%2F

9. Suárez, A. (31 de marzo de 2020). Las clases a distancia durante el confinamiento: transición hacia una educación digital. El diario. Recuperado de:

https://www.eldiario.es/canariasahora/nekuni/bits/confinamiento-transicion-educaciondigital_0_1011749382.html

10. López, P. L. (2004). Población muestra y muestreo. Punto cero, 9(08), 69-74

11. Plan de Centro I.E.S MARÍA ZAMBRANO (septiembre de 2018). I.E.S. María Zambrano. Recuperado de: https:/www.iesmariazambrano.org/images/ultimasnoticias/Curso1819/Organizacion_y_Funcionamiento/PLAN-DE-CENTRO-18-19-def.pdf

12. Gutiérrez-Saldaña, P., Camacho-Calderón, N., \& Martínez-Martínez, M. Lloret, D., Cabrera, V., \& Sanz, Y. (2015). Relaciones entre hábitos de uso de videojuegos, control parental y rendimiento escolar. European journal of investigation in Health, Psychology and education, 3(3), 237-248.

13. Pelechano, V., de Miguel A., \&Pastor, A. (2002). Datos de validación del cuestionario reducido de Locus de Control para Adolescentes (LUCAD-R3). Análisis y modificación de conducta, 28(122), 813-866.

14. Casas, J., Repullo, J. R. \& Donado, J. (2003). La encuesta como técnica de investigación. Elaboración de cuestionarios y tratamiento estadístico de los datos (I). Atención primaria, 31(8), 527-538.

15. Morales, P. (2011). Guía para construir cuestionarios y escalas de actitudes. Universidad Pontificia de Comillas, España.

16. Ortiz, G. (2015). La Encuesta-Diseño de cuestionario II, trabajo de campo y posibles sesgos. Técnicas de Investigación Cuantitativas y Cualitativas.

17. Díaz-Bravo, L., Torruco-García, U., Martínez-Hernández, M., \& Varela-Ruiz, M. (2013). La entrevista, recurso flexible y dinámico. Investigación en educación médica, 2(7), 162-167. 